\title{
Neutropenic enterocolitis associated with Clostridium tertium
}

\author{
N Coleman, G Speirs, J Khan, V Broadbent, D G D Wight, R E Warren
}

\begin{abstract}
A 15 year old boy being treated for relapsed acute lymphoblastic leukaemia developed severe diarrhoea and abdominal pain which worsened despite empirical antibiotic treatment. A right hemicolectomy was performed. The caecum and ascending colon showed changes typical of neutropenic enterocolitis. Clostridium tertium was isolated from faeces, blood cultures, and from the resected gut wall, with no evidence of other organisms capable of causing such a condition. As far as is known, this is the first reported case in which neutropenic enterocolitis has been associated with well documented $C$ tertium infection, an organism previously described as a cause of bacteraemia in neutropenic patients.
\end{abstract}

$(\Im$ Clin Pathol 1993;46:180-183)

Neutropenic enterocolitis is a rare but serious clinicopathological syndrome in which characteristic pathological changes develop in the ileocaecal region of patients with neutropenia, often in association with bacteraemia. ${ }^{1}$ The pathogenesis of the condition is complex, but seems to involve initial mucosal ulceration in the ileocaecal region, with subsequent invasion by clostridia and occasionally other gut organisms. The bacteraemia may lead to septicaemia or metastatic gas gangrene. The production within the intestinal wall of diffusing toxins by the invading organisms is thought to be responsible for the characteristic gut pathology, which is essentially that of severe necrotising oedema of the bowel wall, associated with focal mucosal necrosis, but only scanty infiltration by inflammatory cells.

Clostridium septicum is the usual causal organism $^{2}$ with occasional single case reports of $C$ perfringens, $C$ paraperfringens, $C$ sordellii and $C$ sphenoides. ${ }^{1}$ Other clostridial bacteraemias are described in association with gut signs and symptoms in neutropenic patients, but clinical severity is usually less than with $C$ septicum. Recently a large number of cases of $C$ tertium bacteraemia have been reported. ${ }^{34}$ We present here the first case of $C$ tertium bacteraemia in neutropenia for which a surgical resection specimen was available and which showed histological features of neutropenic enterocolitis.

\section{Case report}

A 15 year old boy, who had developed acute lymphoblastic leukaemia at the age of 11 and had been treated with the UKALL 10 schedule $\mathrm{D}$ protocol developed an isolated testicular relapse, aged 13 . He was reinduced with UKALL 10, with early intensification, testicular irradiation, and maintenance treatment for two years. At the age of 15 he had an isolated central nervous system relapse. $\mathrm{He}$ was reinduced with high dose cytosine and etoposide, together with intrathecal methotrexate, cytosine, and hydrocortisone.

He developed pancytopenia seven days after induction and became pyrexial two days later, developing severe watery diarrhoea mixed with fresh blood, diffuse abdominal pain, and oral mucositis. His C-reactive protein concentration was grossly raised at 212 $\mathrm{mg} / \mathrm{l}$ and blood cultures subsequently grew Pseudomonas aeroginosa and Escherichia coli. Despite initial empirical antibiotic treatment with ceftazidime $(100 \mathrm{mg} / \mathrm{kg} /$ day $)$, gentamicin $(7.5 \mathrm{mg} / \mathrm{kg} /$ day), and metronidazole (30 $\mathrm{mg} / \mathrm{kg} /$ day) he continued to deteriorate clinically, developing severe abdominal pain with melaena. Abdominal $x$ ray pictures showed no intramural gas but there were distended loops of bowel in the right iliac fossa. Further blood cultures on day 4 of antibiotic treatment yielded a pure growth of $C$ tertium. At this stage ceftazidime was replaced by ciprofloxacin (8 mg/kg/day), vancomycin (40 $\mathrm{mg} / \mathrm{kg} /$ day), and oral vancomycin (125 mg four times a day) but despite this he remained pyrexial with worsening abdominal pain.

Further abdominal $x$ ray pictures showed a dilated irregular transverse colon compatible with colitis but no evidence of perforation. An ultrasound scan revealed a thickened transverse colon and free fluid in the pelvis. A computed tomogram of the abdomen showed considerable thickening of the colonic wall, affecting the caecum, ascending colon, hepatic flexure and proximal transverse colon, all of which was compatible with neutropenic enterocolitis. The patient continued to deteriorate clinically, and it was felt that surgical intervention was indicated despite profound neutropenia and thrombocytopenia.

At surgery intraabdominal serous fluid was found, together with an oedematous right colon. There was no evidence of bowel perforation or necrosis macroscopically, but a small area of haemorrhagic necrosis was seen on the posterior surface of the mobilised ascending 
colon. A right hemicolectomy was performed with the formation of an ileostomy. An operative luminal aspirate of the caecum and a piece of gut wall were taken to the bacteriology department within 30 minutes of surgery. Within 24 hours the patient's condition had dramatically improved, and antibiotics were stopped six days after surgery. His ileostomy was closed after one month.

He subsequently underwent autologous bone marrow transplantation after high dose cyclophosphamide and total body irradiation with a cranial boost, and was discharged at six weeks after an uneventful recovery from transplantation.

\section{Pathology}

MACROSCOPIC

The resected bowel was composed of the caecum and ascending colon, which together, were about $25 \mathrm{~cm}$ in length and $10 \mathrm{~cm}$ in diameter (fig 1). The wall of the bowel was thickened and oedematous, producing mucosal folds which bulged into the luminal space. There was patchy mucosal ulceration, together with focal intramural haemorrhage and serosal discoloration.

\section{MICROSCOPIC}

Histological examination showed features characteristic of neutropenic enterocolitis. There was severe transmural oedema, which particularly affected the submucosa of the bowel, where there was also congestion, bleeding, and necrosis (fig 2). The oedema extended into the muscularis propria, where it produced splitting of the muscle fibres. The mucosa was also oedematous, with foci of haemorrhagic surface ulceration. There was no inflammatory cell infiltration. Gram staining showed clusters of Gram positive rods and cocci on the surface of the mucosal ulcers, although no organisms were seen more deeply within the gut wall. Immunofluorescence examination was performed using a mouse monoclonal antibody to $C$ septicum and a rabbit polyclonal antiserum to $C$ tertium, but neither identified organisms. However, cultures of the gut wall performed after alcohol shock yielded $C$ tertium.

\section{Discussion}

$C$ tertium is an aerotolerant clostridium which has been increasingly identified in recent years as a cause of bacteraemia in neutropenic patients, but which is very rare in those without neutropenia. ${ }^{3}$ In previous reports $C$ tertium bacteraemia in neutropenic patients has been associated with signs and symptoms referrable to the gastrointestinal tract or with abdominal cellulitis. The presumed intestinal invasion by $C$ tertium usually causes only relatively mild clinical features, which respond to appropriate antibiotics without surgery, implying that the local damage within the gastrointestinal tract is also relatively mild. To our knowledge this is the first reported case in which histologically demon-
Figure 1 Resected bowel showing bulging, oedematous mucosal folds, with patchy ulceration.

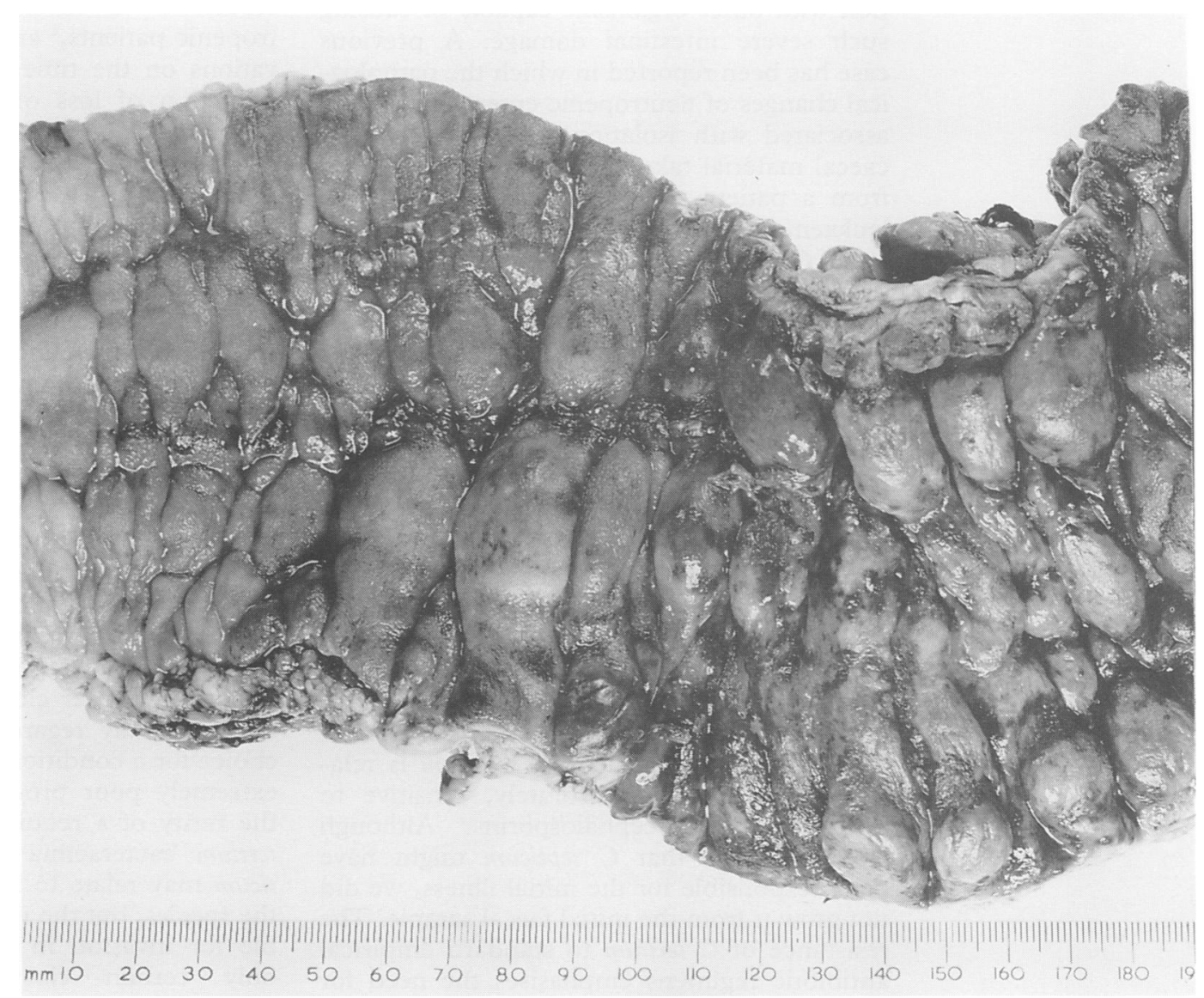


Figure 2 Nectrotising submucosal oedema with overlying mucosal necrosis and haemorrhage.

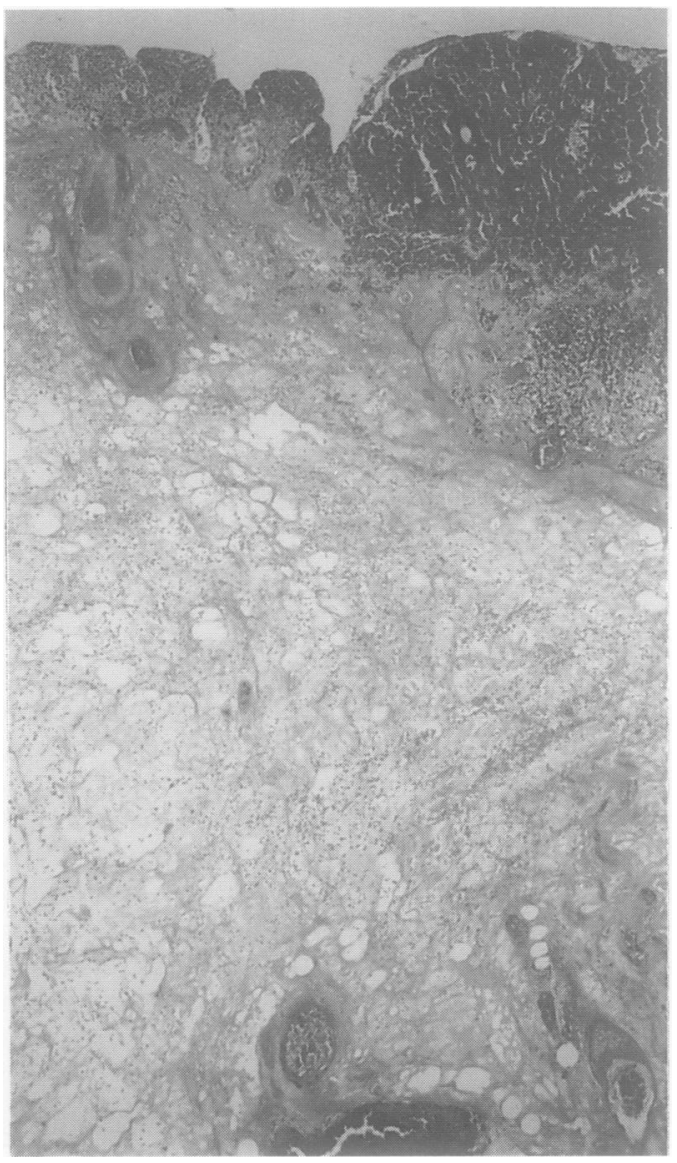

strated neutropenic enterocolitis with gut necrosis has occurred in association with $C$ tertium bacteraemia in the absence of infection with other organisms capable of causing such severe intestinal damage. A previous case has been reported in which the pathological changes of neutropenic enterocolitis were associated with isolation of $C$ tertium from caecal material taken at the time of necropsy from a patient with relapsed acute myeloid leukaemia. ${ }^{5}$ However, the failure to demonstrate $C$ tertium in blood cultures taken before death, together with the frequent presence of this organism as part of the normal faecal flora, ${ }^{3}$ raises doubts as to the importance of finding the isolate after death. In our patient $C$ tertium was isolated from faeces, from blood cultures at the onset of the illness, and from the resected gut wall.

$C$ tertium was detected retrospectively by alcohol shock from faeces at the onset of pyrexia in our patient. The appearance in blood culture on day 4 of antibiotic treatment reflects the ineffectiveness of the chosen initial antibiotic regimen against this organism. Ceftazidime, gentamicin, and metronidazole are inactive under aerobic conditions against this aerotolerant species. ${ }^{3} C$ septicum is relatively more, if not absolutely, sensitive to third generation cephalosporins. ${ }^{6}$ Although we considered that $C$ septicum might have been responsible for the initial illness, we did not grow it from the initial faecal sample. The resistance of $C$ tertium to standard empirical antibiotic regimens emphasises the need for careful speciation of clostridia from blood cultures in neutropenia, and a high index of suspicion in infective episodes with gut symptoms.

$C$ tertium is not thought to cause gas gangrene: there is a single case report of its isolation as the sole clostridial species with Staphylococcus aureus. ${ }^{7}$ The organism is neither proteolytic, lipolytic, haemolytic nor fibrinolytic, nor is it described as producing other toxins or proved virulence factors. The mechanism by which it could produce gastrointestinal myonecrosis is therefore obscure. It is not described as producing the major lethal virulence factor of $C$ septicum-the $a$ toxin nor the $\beta$ toxin of $C$ perfringens, which seems to be the major pathogenic factor in enteritis necroticans and pig-bel. ${ }^{8}$ These virulence factors were not specifically sought in our strain.

Various mechanisms of damage to the ileocaecal mucosa have been described in previous reports of neutropenic enterocolitis, including leukaemic infiltration, intramural haemorrhage, instrumentation related trauma, and ischaemic colitis. ${ }^{1}$ The most likely cause of the initial mucosal damage in this case seems to be treatment with combination antineoplastic drugs which were used to treat our patient's leukaemic relapse. High dose cytosine arabinoside is well known to cause a variety of abnormalities in rapidly proliferating epithelia which range in severity from cytological atypia to frank mucosal ulceration. ${ }^{9}$ The period between the start of the antineoplastic treatment and the isolation of $C$ tertium from blood cultures was 13 days, an interval similar to that seen in previous studies of $C$ tertium bacteraemia in neutropenic patients, ${ }^{4}$ and consistent with observations on the time period required for the induction of loss of mucosal continuity by chemotherapeutic agents. ${ }^{9}$

Once suspected, diagnosis and management of clostridial caecitis can be difficult. ${ }^{10}$ Plain radiography may show dilated loops of large bowel with pneumatosis intestalis, but has a high false negative rate. Characteristic echogenic thickening of the mucosa may be seen in an ultrasound scan and was present in our patient. Computed tomography shows that neutropenic colitis is characterised by caecal wall thickening, with or without pneumatosis, and intraluminal areas of lower density, reflecting areas of oedema or haemorrhage. Most would favour this as the best method of diagnosis: it confirmed the clinical diagnosis in our patient.

The dramatic clinical improvement after surgery is well reported, and early surgical intervention after close clinical monitoring is now generally regarded as the treatment of choice for a condition which otherwise has an extremely poor prognosis. ${ }^{10}$ The reason for the rarity of a requirement for surgery in $C$ tertium bacteraemia ${ }^{3}$ compared with $C$ septicum may relate to intrinsic pathogenicity of the species, but the virulence factors responsible for invasion in neutropenic patients by only certain clostridial species remain unknown. 
1 Newbold KM. Neutropenic enterocolitis. Clin Pathol Rev Dig Dis 1989;7:281-7.

2 King A, Rampling A, Wight DGD, Warren RE. Neutropenic enterocolitis due to Clostridium septicum infection 7 Clin Pathol 1984;37:335-43.

3 Speirs G, Warren RE, Rampling A. Clostridium tertium septicaemia in patients with neutropenia. I Infect Dis septicaemia in patient

4 Valtonen $M$, Sivonen A, Elonen E. A cluster of seven cases of Clostridium tertium septicaemia in neutropenic patients. Eur F Clin Microbiol Infect Dis 1990; 1:40-2.

5 Yates P, MacGowan AP, Potter M, White H, Slade RR. Clostridia and neutropenic enterocolitis. Lancet 1988; i: 185 .
6 Gabay EL, Rolfe RD, Finegold SM. Susceptibility , of Clostridium septicum to 23 antimicrobial agents. Antimicrob Agents Chemother 1981;20:852-3.

7 Masella T. A case of gas gangrene due to Bacillus tertius associated with Staphylococcus aureus. Riforma Med associated with

8 Lawrence G, Skerman F, Freestone DS, Walker PD. Prevention of necrotising enteritis in Papua New Guinea by active immunisation. Lancet 1979; i:227-9.

9 Slavin RE, Dias MA, Saral R. Cytosine arabinoside induced gastrointestinal toxic alterations in sequential chemotherapeutic protocols. Cancer 1978;42:1747-59.

10 Koea JB, Shaw JHF. Surgical management of neutropenic enterocolitis. Bn ₹ Surg 1989;76:821-4.

\title{
Mixed adenocarcinoma/carcinoid tumour of large bowel in a patient with Crohn's disease
}

\author{
Y L Hock, K W M Scott, R H Grace
}

Department of Y L Hock

K W M Scott

Department of

Surgery

R H Grace

The Royal Hospital, Cleveland Road, Wolverhampton WV2 1BT

Correspondence to: Dr YL Hock, Department of Histopathology, Dudley Road Hospital, Dudley Road, Birmingham B18 7QH

Accepted for publication 24 September 1992 Histopathology

\section{Abstract}

A 50 year old woman with a 20 year history of Crohn's disease underwent laparotomy which revealed extensive disease in the small and large bowel, and this was resected. Gross examination of the resected bowel showed features of Crohn's disease as well as a polypoid tumour in the caecum. Histopathological examination of the tumour showed it to be an infiltrating mixed adenocarcinoma/carcinoid tumour arising in a tubulovillous adenoma.

Random sampling of the rest of the bowel affected by Crohn's disease also showed a focus of dysplasia and adeno-



Figure 1 A focus showing typical features of moderately differentiated colonic adenocarcinoma (haematoxylin and eosin). matous change. It is suggested that Crohn's disease may have played a part in the pathogenesis of the tumour.

\section{$(f$ Clin Pathol 1993;46:183-185)}

It has long been recognised that patients with ulcerative colitis may develop large bowel cancer $^{1}$; it has more recently also been recognised that in Crohn's disease there may be malignant change in association with both small and large bowel disease. ${ }^{2}$ Carcinoid tumours have been described both in ulcerative colitis ${ }^{3}$ and Crohn's disease. ${ }^{2}$ Mixed adenocarcinoma/carcinoid tumours have been described in ulcerative colitis ${ }^{1}$ but not in association with Crohn's disease.

\section{Case report}

A 50 year old woman with a 20 year history of small and large bowel Crohn's disease was admitted to hospital for laparotomy following the failure of medical treatment for increasingly severe right iliac fossa pain. There were no clinical features suggestive of a carcinoid syndrome and as there was no clinical suspicion, biochemical tests for carcinoid syndrome were not performed.

Laparotomy showed Crohn's disease from $15 \mathrm{~cm}$ proximal to the ileo-caecal valve to the junction of the descending and sigmoid colon. The region of the appendix was adherent to the anterior abdominal wall. Resection of the affected bowel with ileo-sigmoid anastomosis was performed.

\section{Pathological examination}

The specimen included $15 \mathrm{~cm}$ of terminal ileum and $45 \mathrm{~cm}$ of large bowel. A paracaecal abscess encasing the appendix was noted. On opening the bowel, there was a continuous segment of mucosa showing the typical "cobble stone" appearance of Crohn's disease, measuring $32 \mathrm{~cm}$ in length and involving the 